\title{
DESENVOLVIMENTO DE UM SISTEMA DE ALERTA PARA PREVENÇÃO DE QUEDAS EM PACIENTES HOSPITALIZADOS
}

Heimar F. Marin*

Patricia Bourie**

Charles Safran**

MARIN, H.F.; BOURIE, P.; SAFRAN, C. Desenvolvimento de um sistema de alerta para prevenção de quedas em pacientes hospitalizados. Rev.latino-am.enfermagem, Ribeirão Preto, v. 8, n. 3, p. 27-32, julho 2000.

Este estudo descreve a análise de um Sistema computadorizado de avaliação do paciente no desenvolvimento de um sistema de alerta para prevenir quedas de pacientes hospitalizados. Foi realizada a revisão de prontuários para identificar os fatores de risco presentes no referido instrumento de avaliação. As variáveis identificadas foram: estado funcional do paciente incluindo andar, transferir-se e toilete, sexo e habilidade de realizar auto-cuidado. Usando tais variáveis, foi desenvolvido um sistema de alerta que encontra-se disponibilizado desde Fevereiro de 1997. Em uma segunda etapa, pretende-se estudar o impacto deste sistema na qualidade do atendimento de enfermagem.

UNITERMOS: informática clínica, enfermagem, quedas

\section{INTRODUÇÃO}

Queda de paciente internados é um importante tema de investigação em saúde, estimulando pesquisadores de diversos países a identificar os principais fatores de risco, incidência e conseqüências.

No ambiente hospitalar, conforme ressaltado por TACK et al. (1987) queda de pacientes pode tanto aumentar o tempo de internação, aumentar o custo do tratamento e causar desconforto ao paciente, quanto acarretar septicismo com relação a qualidade do serviço de enfermagem e a responsabilidade do profissional.

Em pessoas idosas, a ocorrência de quedas é também bastante comum, afetando mais de um terço destas pessoas anualmente (TIDEIKSAAR, 1992). Na Finlândia, segundo citação de NURMI et al. (1996), quedas também representam o mais freqüente acidente entre os mais idosos.

Em 1983, o Conselho Nacional de Segurança nos Estados Unidos registrou a ocorrência de quedas como uma causa líder de acidentes fatais em pessoas com idade acima de 74 anos (NIE et al., 1983). Em 1984, mais de 680.000 quedas de pacientes foram relatadas nos hospitais norte-americanos (PARSONS, 1986).

$\mathrm{O}$ custo direto e indireto relacionado a quedas nos Estados Unidos, representa cerca de US\$75 billhões a US\$100 bilhões de dólares a cada ano (URTON, 1991). Além dos custos mensuráveis, vale ressaltar que as fraturas ósseas, os traumatismos e demais injúrias provenientes das quedas, podem limitar um indivíduo na sua atividade e independência, comprometendo o bemestar físico e mental.

A literatura apresenta várias definições para quedas, quase todas semelhantes. DUTHIE (1989) cita como referência a definição de Tinetti, na qual "uma queda é uma situação intencional, onde o indivíduo vai para o chão ou para um nível mais baixo de superfície, não como resultado de um evento instrínseco maior (por exemplo, uma síncope) ou um inevitável perigo que pode resultar em uma queda também para pessoas mais jovens e saudáveis".

Para que os gastos, injúrias e danos indesejáveis aos pacientes e profissionais sejam evitados, medidas preventivas precisam ser adotadas a curto prazo, na tentativa de reverter este processo. Porém, na adoção de procedimentos que possam eliminar ou diminuir o risco de um determinado indivíduo para quedas, faz-se necessário o conhecimento prévio de quais indivíduos são mais propensos e possuem maior chance.

LAWRENCE \& MAHER (1992) definem queda como um escorregão não planejado para o chão, com presença ou não de injúria.

\footnotetext{
* Professor Adjunto, Universidade Federal de São Paulo, pós-doutorado em Informática Médica na Harvard Medical School, com apoio FAPESP

** Center for Clinical Computing, Harvard Medical School, USA
} 


\section{OBJETIVO}

O objetivo deste estudo foi identificar variáveis que pudessem predizer o risco que um paciente possui ou não para sofrer uma queda durante o tempo de internação hospitalar, utilizando o sistema computacional já implantado.

\section{LOCAL DE ESTUDO}

O hospital onde o estudo foi realizado, utiliza para avaliação dos pacientes no momento da internação, um sistema computacional que foi desenvolvido pelo Departamento de Enfermagem e Centro de Computação Clínica da Harvard Medical School, em Boston, USA (SAFRAN et al.,1989).

Este hospital caracteriza-se como hospital de ensino, possuindo um total de 450 leitos, faz cerca de 29.000 admissões e 300.000 consultas ambulatoriais por ano. O sistema de Avaliação de Enfermagem é baseado no modelo de Marjorie GORDON (1994), Functional Health Patterns (Avaliação dos Padrões Funcionais de Saúde), que tem sido a referência da prática de enfermagem desde 1981. Este sistema computadorizado está implantado desde 1994 em todas as enfermarias e na pré-admissão cirúrgica. A sua utilização tem possibilitado a realização de aproximadamente 2.000 avaliações na forma "on-line" a cada mês (BOURIE et al., 1997).

\section{VARIÁVEIS DE ESTUDO}

Embora diferentes estudos identificam e demonstram o grau de predição de determinados fatores de risco, este especificamente, buscou, dentro do instrumento de avaliação do paciente feito pelos enfermeiros no momento da internação hospitalar, quais variáveis pudessem predizer tal risco.

A literatura mostra vários estudos analisando diferentes variáveis que podem predizer as chances de um paciente com risco maior para quedas. Dentre elas, podemos citar como exemplo: idade, diagnóstico médico, sexo, capacidade visual, equilíbrio, história de quedas anteriores, medicação em uso, vertigem e outros.

Dentre todas as mencionadas acima, analisou-se as que se encontram presentes no instrumento de Avaliação de Enfermagem, sendo selecionadas as seguintes: idade, sexo, estado mental do paciente (estado mental), capacidade em andar (andar), capacidade em transferir-se de um local para outro (transferir-se), ir ao toilete e realizar higiene pessoal (toilete), capacidade de cuidar de si mesmo quando receber alta hospitalar (autocuidado).

Uma vez identificadas as variáveis, o sistema computacional então atualizado, fornecerá um aviso, alertando o profissional quando um determinado paciente apresenta uma chance maior para quedas durante a internação hospitalar.

Com isso, espera-se contribuir para que medidas preventivas sejam tomadas em tempo hábil. Também vale mais uma vez enfatizar que a intenção foi usar o mesmo protocolo de coleta de dados, sem modificações aparentes para o usuário, não exigindo treinamento e consumo extra de tempo do enfermeiro para o preenchimento do instrumento de avaliação.

\section{MATERIAL E MÉTODO}

O presente estudo caracteriza-se por ser uma análise retrospectiva, comparando prontuários de pacientes que tiveram quedas durante o período de internação com uma amostra randômica de pacientes que não sofreram quedas e estavam internados no mesmo período, no mesmo hospital.

A amostra consistiu de 389 pacientes. Destes, 193 pertencem ao grupo de pacientes que sofreram queda durante a internação hospitalar e 196 pertencem ao grupo controle.

O período de coleta de dados se deu de 01 de janeiro a 30 de junho de 1996. Os dados foram obtidos dos prontuários dos pacientes arquivados e disponibilizados no sistema computacional. No caso dos pacientes que sofreram queda, informações adicionais foram obtidas por meio de relatório de ocorrência que é preenchido pelos enfermeiros. Não se considerou a presença ou não de trauma e injúria pós-queda para efeito de inclusão na amostra.

Para identificar as variáveis significantes que seriam usadas para desenvolver o sistema de aviso ao profissional de enfermagem responsável pelos pacientes com maior chance de sofrerem queda durante a internação hospitalar, cada variável selecionada foi dicotomizada e estudada separadamente.

Para análise dos dados, utilizou-se o teste Quiquadrado como teste de significância não paramétrico. Os dados foram estruturados em Banco de Dados e analisados com o uso de um software estatístico, o BMDP - Statistical Software, desenvolvido pela VC Press, Los Angeles, Califórnia (1983).

\section{RESULTADOS E DISCUSSÃO}

Dos 389 pacientes selecionados, 214 (55,01\%) 
tinham 65 anos de idade ou mais, conforme mostra a Tabela 1. Destes, 117 (54,67\%) sofreram queda durante o período de internação hospitalar.

Tabela 1 - Distribuição dos pacientes segundo a idade - 1996

\begin{tabular}{ccccccc}
\hline Idade & \multicolumn{2}{c}{ Controle } & \multicolumn{2}{c}{ Estudo } & \multicolumn{2}{c}{ Total } \\
& $\mathrm{N}$ & $\%$ & $\mathrm{~N}$ & $\%$ & $\mathrm{~N}$ & $\%$ \\
\hline$<65$ anos & 99 & 56,57 & 76 & 43,43 & 175 & 100,00 \\
$\geq 65$ anos & 97 & 45,33 & 117 & 54,67 & 214 & 100,00 \\
\hline \multirow{2}{*}{ Total } & \multirow{2}{*}{196} & 50,39 & 193 & 49,61 & 389 & 100,00 \\
\hline
\end{tabular}

De acordo com JANKEN et al. (1986), apesar da afirmação de que pacientes com risco para queda podem ser facilmente identificados, poucos estudos científicos têm demonstrado fatores de risco em comum. WALKER \& HOWLAND (1991) relataram que pessoas com 65 anos ou mais sofrem quedas pelo menos uma vez ao ano, em média.

$\mathrm{Na}$ amostra estudada, a variável idade mostrouse significante $(p=0.02)$, devendo portanto, ser considerada como um dos valores preditivos para identificação dos pacientes sujeitos à queda no período de internação hospitalar.

Com relação ao sexo, na amostra, 50,9\% dos pacientes eram do sexo masculino (Tabela 2).

Tabela 2 - Distribuição da população estudada segundo o sexo - 1996

\begin{tabular}{crrrrrr}
\hline \multirow{2}{*}{ Sexo } & \multicolumn{2}{c}{ Controle } & \multicolumn{2}{c}{ Estudo } & \multicolumn{2}{c}{ Total } \\
& N & $\%$ & \multicolumn{1}{c}{ N } & $\%$ & \multicolumn{1}{c}{ N } & $\%$ \\
\hline Masculino & 108 & 54,55 & 90 & 45,45 & 198 & 100,00 \\
Feminino & 88 & 46,07 & 103 & 53,93 & 191 & 100,00 \\
\hline Total & \multirow{2}{*}{196} & 50,39 & 193 & 49,61 & 389 & 100,00 \\
\hline
\end{tabular}

O sexo como variável de predição para quedas, embora apareça em vários estudos, nem sempre pode ser considerado como valor conclusivo. Alguns estudos relatam que existe maior incidência de quedas em homens e outros apresentam o sexo feminino como mais predisponente (BERRY et al., 1981; WILDE et al., 1981). OVERSTALL em 1980, encontrou incidência de $20 \%$ em homens e $40 \%$ em mulheres. Ambos, assim como a presente amostra estudada, demonstram que as mulheres possuem maior chance para quedas.

No momento da internação hospitalar, uma das questões presente na Avaliação de Enfermagem diz respeito ao estado mental do paciente. Esta questão, no referido instrumento usado pelos enfermeiros, é estruturada, contendo as seguintes possibilidades de escolha no menu de opções simples: agitado, alerta, alerta e orientado, comatoso, combativo, confuso, confuso e agitado, confuso e combativo, desorientado, orientado à pessoa, lugar e tempo, ver avaliação cognitivo perceptual e não respondente.

Para fins de análise, a variável foi dicotomizada e assumiu os seguintes valores: alerta e/ou orientado, na qual estão incluídos todos os pacientes em cujo prontuário estava registrado: alerta, alerta e orientado, orientado à pessoa, lugar e tempo. A segunda categoria estabelecida foi "outros", na qual estão os demais achados, como por exemplo, agitado, confuso e comatoso. Ou seja, o paciente, por algum motivo não se encontra alerta e/ou orientado.

A Tabela 3 mostra que dos 389 pacientes, 372 tinham esta informação presente no prontuário. Destes, 345 (92,74\%) estavam em condição alerta e/ou orientado por ocasião da avaliação.

\section{Tabela 3 - Distribuição dos pacientes segundo o estado} mental - 1996

\begin{tabular}{ccccccc}
\hline Estado & \multicolumn{2}{c}{ Controle } & \multicolumn{2}{c}{ Estudo } & \multicolumn{2}{c}{ Total } \\
Mental & N & $\%$ & N & $\%$ & N & $\%$ \\
\hline $\begin{array}{c}\text { Alerta } / \text { ou } \\
\text { orientado } \\
\text { Outro }\end{array}$ & 180 & 52,17 & 165 & 47,83 & 345 & 100,00 \\
\hline Total & 185 & 49,73 & 187 & 50,27 & 372 & 100,00 \\
\hline
\end{tabular}

Obs: 17 pacientes não possuíam a informação registrada nos protuários

Observa-se no entanto que, dos 117 pacientes que sofreram queda durante o período de internação, $81,48 \%$ não estavam incluídos na categoria "alerta e/ou orientado". Ou seja, o estado mental do paciente alterado, dentre outros, pode configurar também um fator de risco para quedas. ROGERS (1994) salienta que o estado mental do paciente também é um dos fatores críticos na identificação de risco para quedas. Em um estudo, observou que $34 \%$ dos pacientes que caíram, tinham estado cognitivo alterado. Similarmente, LAWRENCE \& MAHER (1992) em uma relação entre alteração do estado mental e quedas, encontraram que dos 14 pacientes que sofreram queda, 13 tinham estado mental alterado.

Nas Tabelas 4, 5 e 6 a seguir, são apresentados os dados com relação à independência do paciente em excecutar atividades de caminhar (andar), transferir-se de um local para outro e realizar sua própria toilette, respectivamente.

Estes dados são avaliados também no momento da internação e geralmente revistos a cada dia, fazendo parte do quadro de avaliação da capacidade de realizar atividades da vida diária (ADL - Activity Daily Living). 
Nesta avaliação, os valores possíveis encontrados no sistema computacional são: o paciente é independente, o paciente precisa de assistência, o paciente é dependente. Neste estudo, com a dicotomização das variáveis, a categoria "outro" engloba os pacientes cujos valores registrados foram: necessita de assistência ou é dependente.

Tabela 4 - Distribuição dos pacientes segundo capacidade de andar (ADL) - 1996

\begin{tabular}{crrrrrr}
\hline \multirow{2}{*}{ Andar } & \multicolumn{2}{c}{ Controle } & \multicolumn{2}{c}{ Estudo } & \multicolumn{2}{c}{ Total } \\
& $\mathrm{N}$ & $\%$ & \multicolumn{1}{c}{$\mathrm{N}$} & $\%$ & \multicolumn{1}{c}{$\mathrm{N}$} & $\%$ \\
\hline Independente & 163 & 58,01 & 118 & 41,99 & 281 & 100,00 \\
Outro & 27 & 27,55 & 71 & 72,45 & 98 & 100,00 \\
\hline Total & 190 & 50,13 & 189 & 49,87 & 379 & 100,00 \\
\hline
\end{tabular}

Obs: 10 pacientes não possuíam esta informação registrada no prontuário

Tabela 5 - Distribuição dos pacientes segundo capacidade de transferir-se de um local para outro (ADL) - 1996

\begin{tabular}{crrrrrr}
\hline Transferir-se & \multicolumn{2}{c}{ Controle } & \multicolumn{2}{c}{ Estudo } & \multicolumn{2}{c}{ Total } \\
& N & $\%$ & N & $\%$ & N & $\%$ \\
\hline Independente & 166 & 58,87 & 116 & 41,13 & 282 & 100,00 \\
Outro & 24 & 24,74 & 73 & 75,26 & 97 & 100,00 \\
\hline Total & 190 & 50,13 & 189 & 149,87 & 379 & 100,00
\end{tabular}

Obs: 10 pacientes não possuíam esta informação registrada no prontuário

Tabela 6 - Distribuição dos pacientes segunda capacidade de realizar o próprio toilette (ADL) - 1996

\begin{tabular}{crrrrrr}
\hline Toilette & \multicolumn{2}{c}{ Controle } & \multicolumn{2}{c}{ Estudo } & \multicolumn{2}{c}{ Total } \\
& $\mathrm{N}$ & $\%$ & $\mathrm{~N}$ & $\%$ & $\mathrm{~N}$ & $\%$ \\
\hline Independente & 168 & 57,14 & 126 & 42,86 & 294 & 100,00 \\
Outro & 23 & 27,38 & 61 & 72,62 & 84 & 100,00 \\
\hline Total & 191 & 50,53 & 187 & 49,47 & 378 & 100,00
\end{tabular}

Obs: 11 pacientes não possuíam esta informação registrada no prontuário

As tabelas mostram que os resultados são bastante semelhantes e possuem significante participação na predição de pacientes com chance para queda $(\mathrm{p}=0.00)$.

HALPERT \& CONNORS (1986) em um estudo com 181 pacientes com história de quedas, observaram que 124 estavam alertas e/ou orientados. Porém, destes, $61(49 \%)$ caíram da cama, $22(18 \%)$ caíram enquanto deambulavam, $31(25 \%)$ caíram no caminho para o banheiro e $10(8 \%)$ caíram da cadeira.
Assim, muitas vezes, independente do estado mental do paciente, outros fatores podem contribuir para que a queda ocorra. Outros estudos, como por exemplo o de ROGERS (1994) ressalta que cerca de 30\% das quedas ocorrem quando o paciente sai do leito, vai ao banheiro e mesmo durante o toilette.

Em termos de resultados e de predição para quedas, observa-se então que se o paciente é dependente e/ou precisa de assistência para realizar qualquer uma destas atividades acima descritas, é uma indicação de que tal paciente tem maior chance de sofrer quedas.

Na Tabela 7 estão apresentados os dados relativos a capacidade do paciente em cuidar de si mesmo na ocasião que receber a alta hospitalar. Em termos de assistência de enfermagem, esta avaliação deve determinar ou pelo menos, indicar o grau de necessidade e se um serviço de atendimento domiciliar precisa ser disponibilizado ao paciente após a alta.

No instrumento de Avaliação de Enfermagem, esta questão é estruturada, podendo assumir os seguintes valores: o paciente é capaz de cuidar de si mesmo, o paciente é capaz de cuidar de si mesmo com ajuda, o paciente é incapaz de cuidar de si mesmo.

Para efeito de tratamento, estes dados foram dicotomizados em: "o paciente é capaz de realizar autocuidado" e "o paciente precisa de ajuda ou é incapaz".

Tabela 7 - Distribuição dos pacientes segundo capacidade de auto-cuidado por ocasião da alta hospitalar - 1996

\begin{tabular}{crrrrrr}
\hline \multirow{2}{*}{ Auto-cuidado } & \multicolumn{2}{c}{ Controle } & \multicolumn{2}{c}{ Estudo } & \multicolumn{2}{c}{ Total } \\
& N & $\%$ & N & $\%$ & N & $\%$ \\
\hline É capaz & 122 & 70,52 & 51 & 29,48 & 173 & 100,00 \\
Incapazlajuda & 67 & 33,50 & 133 & 66,50 & 200 & 100,00 \\
\hline Total & 189 & 50,67 & 184 & 49,33 & 373 & 100,00 \\
\hline
\end{tabular}

Obs: 16 pacientes não possuíam a informação registrada no prontuário

Esta variável também se mostrou significante para identificação de paciente com potencial para queda $(\mathrm{p}=0.00)$. Observa-se que dentre os pacientes julgados pelo enfermeiro, que por ocasião da alta hospitalar necessitariam de cuidado domiciliar, 133 (66,50\%), sofreram queda durante o período de internação. Isto mostra que, uma vez o paciente sendo considerado dependente ou com necessidade de assistência na vida diária, esta dependência também se apresenta e precisa ser considerada durante a internação. Assim, intervenções de enfermagem apropriadas precisam ser adotadas para que possam eliminar ou diminuir o risco do paciente sofrer quedas durante a hospitalização. 


\section{DESENVOLVIMENTO DO SISTEMA}

A identificação destas variáveis permitiu desenvolver um sistema computacional de alerta e dispôlo ao profissional no momento em que realiza a internação de um paciente, sendo atualizado assim que novas avaliações de enfermagem são realizadas.

Um sistema de alerta é uma mensagem emitida pelo computador que aparece quando o profissional de saúde (médico e/ou enfermeiro) olha para o registro médico do paciente "on-line" (SAFRAN et al., 1996). Muitos destes sistemas já estão em uso por mais de 6 ou 7 anos nos hospitais norte-americanos, apresentando excelentes resultados na melhoria do atendimento fornecido (KUPERMAN et al.; 1995, SAFRAN et al., 1996).

Neste estudo, especificamente, embora muitos fatores estejam sendo apontados pela literatura como de risco para queda de pacientes, optou-se por, numa primeira etapa, dentro dos recursos em uso e disponíveis aos enfermeiros no hospital, verificar quais variáveis pudessem predizer a chance de queda, e com estas variáveis, estabelecer as regras para o desenvolvimento do sistema, fornecendo o aviso ao profissional em tempo real.

Assim, com as variáveis conhecidas, o Sistema de Enfermagem recebeu algumas regras, não sendo porém, modificado com relação ao conteúdo e estrutura inicial. Portanto, no momento em que o enfermeiro faz a admissão hospitalar do paciente, havendo uma das variáveis presente, ao final da entrevista, uma tela é apresentada ao profissional, com a informação de que o paciente possui risco para queda. Esta tela apresenta ainda a razão pela qual o risco foi identificado e sugere implantação de medidas preventivas cabíveis.

\section{CONSIDERAÇÕES FINAIS}

Este estudo representou um passo inicial para a implantação de um protocolo de medidas preventivas. Conhecendo os pacientes com maior chance para quedas, os profissionais podem adotar específicas medidas preventivas e de segurança, preservando a saúde dos pacientes e a qualidade do atendimento prestado.

O protocolo de intervenções para pacientes com potencial para injúrias também encontra-se disponível no sistema computacional e recomenda que algumas ações sejam adotadas com relação ao paciente. Por exemplo: supervisionar a Avaliação ADL sempre que necessário, dispor itens pessoais ao alcance do paciente, manter o ambiente seguro, colocar o leito em posição mais baixa, mantendo as rodas travadas e grades laterais suspensas, entre outras.

Este sistema atualizado, está na forma "on-line" desde fevereiro de 1997. Assim, este estudo não termina aqui. Em uma segunda etapa, pretende-se analisar as conseqüências deste dispositivo de aviso na consulta do profissional, adotando medidas preventivas e de segurança e no cuidado ao paciente.

\section{Agradecimentos:}

Nosso especial agradecimento ao Dr. Samuel Goihman da Universidade Federal de São Paulo pelo inestimável auxílio na análise estatística e a Michael McKay do Center for Clinical Computing na programação do sistema de alerta.

\section{DEVELOPMENT OF A SYSTEM TO PREVENT INPATIENTS FALLS}

The present study describes the analysis of an online patient assessment system developed to prevent inpatients falls. A chart review was performed in order to identify risk factors present in the Nursing Assessment tool. The identified variables were Functional status including walking, transferring and toilet, sex and patients self-care ability. Authors developed a system using these variables, that is available since February 1997. In a second phase of this study, authors aim to evaluate the effectiveness of this computerized intervention.

KEY WORDS: clinical informatics, nursing, falls

\section{DESARROLLO DE UN SISTEMA DE ALERTA PARA LA PREVENCIÓN DE LAS CAÍdAS EN PACIENTES HOSPITALIZADOS}

Este estudio describe el análisis de un sistema de computación en enfermería para desarrollar un sistema de alerta para prevenir caidas de los hospitalizados. Una revisión de las historias clínicas fue realizada para identificar los factores de riesgo presentes en el referido instrumento de evaluación. Las variables identificadas fueron: estado funcional del paciente incluyendo 
andar, desplazamiento, limpieza, sexo y habilidad para realizar el autocuidado. Usando estas variables un sistema de alerta fue desarrollado e incorporado en el sistema desde febrero de 1997. En una segunda fase de este estudio nos preponemos evaluar la eficacia de esta intervención automatizada en la calidad del cuidado de enfermería..

TÉRMINOS CLAVES: informática clínica, enfermería, accidentes por caídos

\section{REFERÊNCIAS BIBLIOGRÁFICAS}

01. BERRY, G.; FISHER, R.H.; LANG, S. Detrimental incidents, including falls in an eldery institutionalized population. J.Am. Geriatr. Soc., v. 29, n. 7, p. 322- 4, 1981.

02. BOURIE, P.Q.; CHAPMAN, R.H.; DAI, S.; REILEY, P. An automated nursing assessment for a teaching hospital. MD Computing, v. 14, n. 1, p. 57-60, 1997.

03. DUTHIE, E.H. Falls. Med. Clin. North Am., v. 73, n. 6, p. 1321-36, 1989.

04. GORDON, M. Nursing diagnosis, process and application. Mosby: New York, 1994.

05. HALPERT, A.; CONNORS, J.P. Prevention of patient falls through perceived control and other techniques. Law, Medicine \& Health Care, v. 14, n. 1, p. 20-4, 1986.

06. JANKEN, J.K.; REYNOLDS, B.A.; SWIECH, K. Patient falls in the acute care setting: identifying risk factors. Nurs. Res., v. 35, n. 4, p. 215-9, 1986.

07. KUPERMAN, G.J.; TEICH, J.M.; BATES, D.W.; McLATCHEY, J.; HOFF, T.G. Representing hospital events as complex conditionals. In: SYMPOSIUM FOR COMPUTER APPLICATIONS IN MEDICAL CARE (SCAMC). Proceedings. Washington, DC, 1995. p. $137-41$.

08. LAWRENCE, J.I.; MAHER, P.L. An interdisciplinary falls consult team: a collaborative approach to patient falls. J. Nurs. Care, v. 6, n. 3, p. 21-9, 1992.

09. NIE, N.H.; HULL, C.H.; JENKINS, J.G.; STEINBRENNER, K.S.; BEN, D.H. National Safety Council: accidents facts. New York: McGraw-Hill Book, 1983.
10. NURMI, I.; SIHVONEN, M.; KATAJA, M.; LUTHJE, P. Falls among institutionalized eldery: a prospective study in four institutions in Finland. Scand. J. Caring Sci., v. 10, n. 4, p. 212-20, 1996.

11. OVERSTALL, P.W. Prevention of falls in the eldery. J. Am. Geriatr.Soc, n. 28, p. 481-4, 1980.

12. PARSONS, M.J. Five common legal risks. Nurs. Life, v. 6, n. 6, p. 26-30, 1986.

13. ROGERS, S. Reducing falls ina rehabilitation setting: a safer environment through team effort. Rehabil. Nurs., v. 19, n. 5, p. 274-6, 1994.

14. SAFRAN, C.; SLACK, W.V.; BLEICH, H.L. Role of computing in patient care in two hospitals. MD Computing, v. 6, n. 3, p. 149-55, 1989.

15. SAFRAN, C.; RIND, D.M.; DAVIS, R.B.; IVES, D.; SANDS, D.Z.; CURRIER, J.C.; SLACK, W.V.; COTTON, D.J.; MAKADON, H.J. Effects of a knowledge-based eletronic patient record on adherence to practice guidelines. MD Computing, v. 13, n. 1, p. 55-63, 1996.

16. TACK, K.A.; ULRICH, B.; KEHR, C. Patient falls: profile for prevention. J. Neurosci. Nurs., v. 19, n. 2, p. 83-9, 1987.

17. TIDEIKSAAR, R. Falls among the eldery: a community prevention program. Am. J. Public Health, v. 82, n. 6, p. 892-893, 1992.

18. URTON, M.M. A community home inspection approach to preventing falls among the eldery.

Public Health Reports, n. 106, p. 192-5, 1991.

19. WALKER, J.E.; HOWLAND, J. Falls and fear of falling among eldery persons living in the community: occupational therapy interventions. Am. J. Occup. Ther., n. 45, p. 119-22, 1991.

20. WILDE, D.; NOVAR, L.; ISAACS, B. Facts on falling. Health Social Sci. J., v. 91, p. 1413-5, 1981. 